\title{
Secondary osteosarcoma arising on one of multiple areas of avascular bone necrosis caused by corticosteroid therapy: case report and review of the literature
}

\author{
Benedetta Spazzoli*, Giovanna Danna, Roberta Laranga, Marco Gambarotti and Davide Maria Donati \\ Medico Chirurgo, Specialista in Ortopedia e Traumatologia, III clinica ortopedica a prevalente indirizzo oncologico, Istituto Ortopedico Rizzoli, Bologna, Italy
}

\begin{abstract}
Abbreviations: CT: Computerized tomography; n-CT: neoadjuvant chemotherapy; p-CT: post-operative chemotherapy; MFH: malignant fibrous histiocytoma; FS: fibrosarcoma, OS: Osteosarcoma.

\section{Introduction}

The category of secondary osteosarcoma (OS) was introduced by the WHO in their most recent classification of soft tissue and bone tumors (Flanagan AM, Bridge JA, O'Donnel PG. Secondary osteosarcoma in WHO Classification of Soft Tissue and Bone Tumours [Fifth Edition, LYON:IARC (International Agency for Research on Cancer), p. 419-421 is defined as an OS arising in abnormal bone and includes OS in Paget disease of bone [1], radiation-associated sarcoma [2], infarct-related OS [3], OS due to chronic osteomyielitis [4] and OS secondary related to early postzygotic disorders such as fibrous dysplasia [5].
\end{abstract}

A peculiar characteristic of secondary OS is that it tends to manifest in a population older than that of primary OS, which, conversely, is more common in younger population [3]. This has implications on the overall management, because older patients could not be eligible for standard chemotherapy or could not undergo the expected number of courses because of hepatic or renal toxicity or, otherwise, they may present more side effects when on these drugs.

As bone infarcts are usually asymptomatic, their presence in the general population is unknown. A major part of our knowledge derives from incidental discovery via findings from radiographs. Although a variety of conditions are associated with bone infarct such as alcoholism, the use of pharmacological corticosteroids, sickle-cell anemia, previous radiotherapy, and Caisson's disease [6]; furthermore, many patients do not have any evident causes. Also called avascular bone necrosis areas, bone infarcts can present at any skeletal site; they are most commonly found at the distal femur, proximal tibia and proximal femur [6]. The presence of sarcoma in association with bone infarct is rare. Although Johnson referred to a similar event in 1953. The first documented case was reported by Furey et al. in 1966 and it was a fibrosarcoma [3].

In existing literature, McDonald in 2018 present a case of OS on bone infarct and analyze all known cases prior to publication, for a total of 15 cases [7].

We present a case of a bone infarct-related OS in a 66-year-old male with multiple areas of avascular bone necrosis. Here we describe the clinical, radiological, and histological features of this patient, and present a review of the literature.

\section{Case report}

A 66-year-old male was referred to our hospital with a threemonths history of pain and increasing swelling on the left thigh and knee, without prior trauma.

Forty-two years before, this patient received a diagnosis of idiopathic medullary aplasia, for which he received steroid therapy, with subsequent development of multiple areas of bone necrosis. After 28 transfusions, hematologists performed a therapy with anti-lymphocytic globulin and high-dose bolus methylprednisolone, ultimately leading to the complete resolution of bone marrow disease.

The patient arrived at our hospital for the presence of pain and swelling on the left thigh, a bilateral MRI and computerized tomography CT-scan of the knee were performed, revealing typical map lesions of bone necrosis in both distal femurs and proximal tibias. Additionally, in the left thigh, a mass adjacent to the necrosis with destruction of anterior and posterior cortices and subsequent leakage of heteroplastic tissue out of the bone was evident.

Orthopedics, elsewhere, also performed incisional biopsy of the mass, with a histological diagnosis of pleomorphic sarcoma. No parenchymal lesions were evident upon CT-scan of the chest. Totalbody PET showed uptake on distal left femur (SUV 31). The patient started neoadjuvant chemotherapy with Epirubicin and Ifosfamide.

Laboratory exams before surgery showed normal RCP $(0,25$ on $0,5)$ and LDH (225 on 248) levels, as well as increased VES (39 on 20) and ALP (263 on 120). The patient had mild pancytopenia due to chemotherapy.

The patient was treated with three courses of pre-operative chemotherapy: at the end of the second course, he had pathological fracture making a movement lying in bed. A knee brace locked in extension has been placed during the last chemotherapy course; meanwhile he developed external sciatic popliteal nerve palsy. Limb salvage surgery with distal femur resection and mega-prosthesis was performed at the end of preoperative chemotherapy.

${ }^{*}$ Correspondence to: Benedetta Spazzoli, Medico Chirurgo, Specialista in Ortopedia e Traumatologia, III clinica ortopedica a prevalente indirizzo oncologico, Istituto Ortopedico Rizzoli, Bologna, Italy, E-mail: benedettaspazzoli@gmail.com

Key words: Secondary osteosarcoma, bone infarct, bone tumor

Received: March 05, 2021; Accepted: March 12, 2021; Published: March 15, 2021 
Histological examination of the specimen revealed a lesion measuring $14 \times 6 \times 6 \mathrm{~cm}$., that histologically corresponded to extensively necrotic high-grade osteoblastic OS, (95\% of necrosis, grade 3 according to Huvos) [8]. Surgical margins were wide.

After 6 months, periprosthetic joint (PJI) infection occurred; firstly, it was treated with irrigation and debridement, nonspecific antibiotics (intraoperative cultural exams not performed) and VAC-therapy. This approach was not successful, RCP was always out of range, sinus still present, thigh and knee warm and painful, so, two months later, he underwent explant of the prosthesis and vancomycin-added cement spacer. Intraoperative histological examination showed chronic and acute inflammation, Pseudomonas Aeruginosa was detected in all intraoperative cultural exams; therefore, specific oral antibiotic therapy was administered.

Every twenty days, the patient performed laboratory blood exams for RPC and VES, in order to check the inflammatory status. RCP and VES were normal in every examination, so, after sixty-nine days after the positioning of the spacer, a silver-coated prosthesis in arthrodesis was positioned, after removal of antibiotic-added cement spacer. Intraoperative cultural exams were negative.

$\mathrm{X}$-ray films of left thigh and knee at 40 days after last surgery showed no mechanical complications. At the last follow up, 12 months after first surgery, there was no evidence of local recurrence or metastases.

\section{Discussion}

Secondary bone infarct-related OSs represent about $1 \%$ of all osteosarcomas [7]. In most cases, bone infarcts are occasional findings on X-ray films, since they are asymptomatic lesions. Their exact etiology is unknown. They can be idiopathic, or related to prior trauma, alcoholism, hematologic disorders such as sickle cells disease, and chronic use of steroids. Torres and Kyriakos in 1992 described 4 patients with OS on bone infarct in multiple areas of bone avascular necrosis, but they did not specify any information about the etiopathology of the multiple bone infarcts [3].

Here we reported a case in which the origin of bone infarct is known and documented.

Different types of sarcomas are able to grow on top of a bone infarction, with the most common being undifferentiated pleomorphic sarcoma (UPS) at $63 \%$, followed by OS at $18 \%$ and fibrosarcoma (FS) at $(13,2 \%)[1]$.

Secondary bone infarct-related OS generally affects the older-aged population more frequently than conventional osteosarcoma [1]. In the literature, the mean age is 57-year-old; two-thirds of patients are male [6]. The time interval between bone infarct and sarcoma development is unknown, as the infarct is asymptomatic. Torres et al. [3] found that the time interval from last exposure to compressed air conditions, thought to be the timing and cause of bone infarct, was many years, in some cases more than 20. A slow and steady reparative stimulus on infarct area, could achieve the growth of OS above it as final result, which is coherent with the idea that secondary OS affects populations of older ages compared to those with primary OS.

Bone infarct-related OSs most frequently arise in the distal femur, proximal tibia, and proximal femur, while they are rarely seen in the humerus [7].

Most patients presented with pain and swelling in the area of bone infarct, sometimes with pathological fracture.
The relationship between the development of OS and an underlying bone infarct is currently unknown. One hypothesis involves a possible excessive proliferative activity of the reparative cells around damaged areas of the bone infarct. Mirra et al. [9] suggested that sarcomas arise not directly inside the infarct but rather in continuity with them. This alludes a causal relationship, with the cause, being the bone infarct, and the effect, being the reparative cells near and around the infarct, proliferating in order to compensate for the loss of healthy tissue. Johnson et al. [10] stated that excessive cell proliferation is likely to lead to neoplasia and described the "actively proliferating margin" of a bone infarct and its progression to a sarcoma, as an example. Dorfman et al. further corroborated this hypothesis by demonstrating that bone infarcts show evidence of reparative and regenerative attempts of a "high degree", and that "excessive proliferative activity" was the basis for the development of sarcoma. Bahk [9] et al described a transition zone that exists in space between the infarct and the sarcoma: this zone is comprised of granulation tissues including interlacing fascicles of spindle cells, capillary proliferation with foamy macrophage, chronic inflammatory cells with cellular atypia of varying degrees.

They concluded, from histological observations, that the transition from granuloma to sarcoma observed in the transitional zone between inert bone infarct and bone sarcoma, could support the idea that infarct-related sarcomas arise from proliferation of reparatory tissues at the periphery of ancient infarcts. However, because no histological evidence has been published regarding the reparative tissue of infarcts as a potential resource of sarcomatous transformation, there is disagreement from other authors.

Torres et al. [3] in 1992 discussed about the cessation of the reparative process, arguing that the proliferative activity could not be the cause of the sarcoma. As for the cause of cessation, it is unknown, but they showed that when a sufficient portion of the infarct has been repaired, the impetus for continued repair process may stop. Another possible reason for cessation could be related with increasingly fibrous, calcified and ossified focus of necrosis, where the capillary vascular proliferation is unable to proceed (Table 1,2).

There are no studies yet that suggest that the adjacent normal marrow tissue continues to proliferate to replace the infarct.

During the last 20 years, the introduction of chemotherapy has changed the approach to OS. Historically, OSs were treated with amputation, and often the patients developed subsequent lung metastases, with short life expectancies [5]. Nowadays, standard treatment is neoadjuvant chemotherapy, followed by limb salvage surgery, and adjuvant chemotherapy. Depending upon the grade of necrosis of the resected specimen, status of the surgical margins, and the presence of metastases, the type of adjuvant chemotherapy is chosen. Survival rate with surgery alone (amputation or resection) went from $11 \%$ to $24 \%[2,10]$ compared to $63 \%$ for patient who received additional chemotherapy $[7,11,12]$.

We analyzed type of treatment and overall survival of fifteen patients with OS on bone infarct described in the literature.

Site of the tumor was femur in 8 patients, proximal tibia in 4 and humerus in the last 3 . Among nine patients who underwent only surgery ( 5 amputations/4 resections), two patients were lost during the follow up; above this group, six patients died of disease in a median time of 12, months (5-40) from diagnosis and one patient survived (48 months). Two patients underwent surgery (amputation) along with radiotherapy, and both of these cases died of the disease in 9 and 
Table 1. Literature series of OS on bone infarct

\begin{tabular}{|c|c|c|c|c|c|}
\hline Case & Age & Sex & Site & Treatment & Course \\
\hline 1 - Johnson (1962) & 48 & M & Proximal Tibia & XRT; Amputation & Lung METs at 3 month; Died at 9 mo. \\
\hline 2 - Mirra (1974) & 61 & M & Distal Femur & XRT; Amputation & Lung METs at 1 month; Died at 3 mo. \\
\hline 3 - Dahin \& Unni (1986) & 79 & M & Proximal Femur & Resection & Died with lung METs at $19 \mathrm{mo}$. \\
\hline 4 - Dahlin \& Unni (1986) & 56 & $\mathrm{~F}$ & Proximal Femur & Amputation & Lung MTEs treated with Chemotherapy; Died at 40 mo. \\
\hline 5 - Heater \& Collins (1987) & 35 & M & Proximal Femur & Amputation; Adjuvant CHT & Bone MET at 6 years treated with amputation; Alive at 57 months \\
\hline 6 - Heater \& Collins (1987) & 61 & $\mathrm{~F}$ & Proximal Tibia & Amputation & Chest and spinal canal METs at 12 mo.; Died few weeks after \\
\hline 7 - Torres and Kyriakos 1992 & 82 & M & Proximal Humours & Amputation & Died with lung METs at $12 \mathrm{mo}$. \\
\hline 8 - Resnik et. Al 1993 & 56 & M & Proximal tibia & Resection & Not Reported \\
\hline 9 - Desai et al. 1996 & 35 & $\mathrm{~F}$ & Distal & Amputation & Dead at 24 mo. \\
\hline 10 - Domson et al. 2009 & 55 & M & Proximal Tibia & Neo-adjuvant CHT; Amputation & Alive at $58 \mathrm{mo}$. \\
\hline 11- Domson et al. 2009 & 75 & $\mathrm{~F}$ & Distal Femur & Resection & Died at 5 mo. \\
\hline 12 - Domson et al. 2009 & 35 & M & Proximal Femur & Palliative XRT & Lost to follow-up less than 1 mo. after \\
\hline 13 - Bahk et al. 2010 & 61 & M & Distal Femur & Amputation & Dead at $11 \mathrm{mo}$. \\
\hline 14 - Endo et al. 2013 & 36 & $\mathrm{~F}$ & Proximal Numerus & Resection & Alive at 4 years. \\
\hline 15 - McDonald et al. 2018 & 72 & M & Proximal Numerus & Neo-adjuvant CHT; Amputation & Died at $7 \mathrm{mo}$. \\
\hline
\end{tabular}

$\mathrm{XRT}=$ radiotherapy; $\mathrm{CHT}=$ chemotherapy MET/METs = metastasis/metastases; mo. $=$ months

Table 2. Overall Survival estimates at different time point of follow up

\begin{tabular}{|c|c|c|}
\hline & \multicolumn{2}{|c|}{ Literature cohort } \\
\hline Time & Outcome & \\
\hline $\mathbf{1 2 - O S}$ & 0.54 & $25-76$ \\
\hline $\mathbf{3 6 - O S}$ & 0.36 & Dec-61 \\
\hline Median survival time & 19 months & 7 -not reached \\
\hline
\end{tabular}

3 months respectively. One patient underwent only radiotherapy and after two weeks he got lost to follow-up. Three, underwent surgery (amputation) as well as chemotherapy, two in the preoperative period, one postoperatively. Two of them were alive without evidence of disease at fifty-eight and fifty-seven months after surgery respectively, while one died of the disease at seven months (Figure 1-3).

Complete follow-up date are available for 12 patients, nine of them died of the disease (75\%) Overall survival at 36 months was 36\% (95\% CI 25-76). Three patients survived without disease, two of them were those who underwent not only surgery, but more importantly chemotherapy in association, one preoperatively, the last postoperatively.

The patient presented in this report, has been treated with three standard preoperative chemotherapy courses, followed by surgical resection and megaprosthesis. He got into early PJI and resolution was obtained with surgical debridement, removal of the prosthesis and antibiotic-added cement spacer. At the normalization of inflammatory blood index, the spacer was removed, and silver-coated prosthesis was placed. At the last follow up, 12 months after distal femur resection, the patient was alive with no evidence of local recurrence neither distant metastases. Up to now, follow up examinations were performed every 3 months with X-ray film of left thigh and knee and CT-scan without contrast of the chest.

\section{Conclusions}

Patients with secondary bone infarct-related OSs have the best chances of survival with chemotherapy, combined with surgery. When possible neo-adjuvant chemotherapy, by shrinking tumour dimensions, limb salvage surgery became more possible rather than amputation. However, regardless of the type of local treatment, poor prognosis is related to development of lung metastases after surgery, and this confirms the key role of chemotherapy courses to deal with the treatment of this systemic disease.

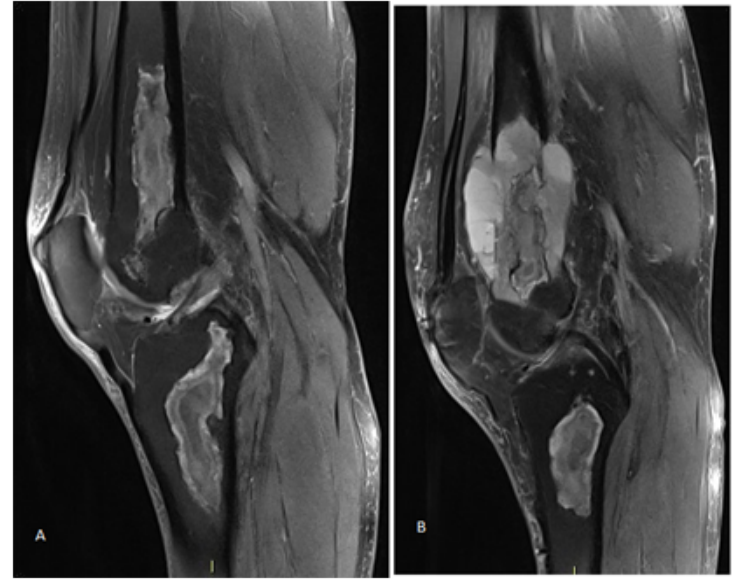

Figure 1. MRI showed multiple bilateral bone infarcts around right and left knee, on the left distal femur necrotic area, heteroplastic tissue grown with rupture of cortices and soft tissue expansion

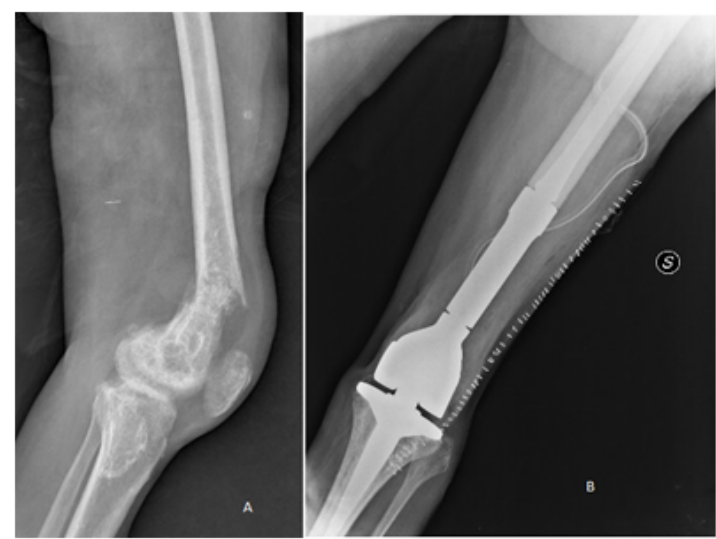

Figure 2. Pathological fracture of left distal femur and postoperative X-ray film of megaprosthesis reconstruction 


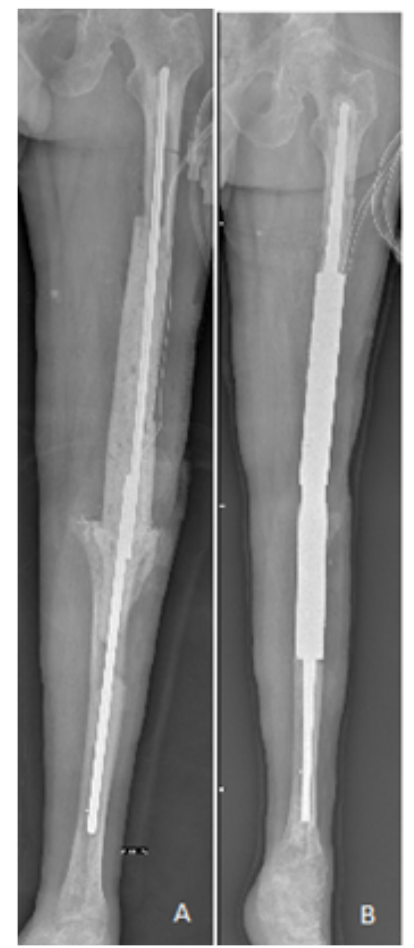

Figure 3. Antibiotics-added cement spacer in arthrodesis and silver-coated prosthesis in arthrodesis after removal of the spacer

\section{References}

1. Mangham DC, Davie MW, Grimer RJ (2009) Sarcoma arising in Paget's disease of bone: Declining incidence and increasing age at presentation. Bone 44: 431-436. [Crossref]

2. Lorigan J, Libshitz H, Peuchot M (1989) Radiation-induced sarcoma of bone: CT findings in 19 cases. AJR Am J Roentgenol 153: 791-794. [Crossref]

3. Torres FX, Kyriakos M (1992) Bone infarct-associated osteosarcoma. Cancer 70: 2418-2430. [Crossref]

4. Puri A, Parasnis AS, Udupa KV, Duggal A, Agarwal MG (2003) Fibroblastic Osteosarcoma Arising in Chronic Osteomyelitis. Clin Radiol 58: 170-172. [Crossref]

5. Sugiura Y, Kanda H, Motoi N, Nomura K, Inamura K, et al. (2018) Osteosarcoma arising in fibrous dysplasia, confirmed by mutational analysis of GNAS gene. Pathol Res Pract 214: 318-324. [Crossref]

6. Domson GF, Shahlaee A, Reith JD, Bush CH, Gibbs CP (2009) Infarct-associated Bone Sarcomas. Clin Orthop Relat Res 467: 1820-1825. [Crossref]

7. McDonald MD, Sadigh S, Weber KL, Sebro R (2018) A Rare Case of an Osteolytic Bone-infarct-associated Osteosarcoma: Case Report with Radiographic and Histopathologic Correlation, and Literature Review. Cureus. 10: e2777. [Crossref]

8. Huvos AG (1986) Osteogenic sarcoma of bones and soft tissues in older persons. A clinicopathologic analysis of 117 patients older than 60 years. Cancer 57: 1442-1449. [Crossref]

9. Mirra JM, Bullough PG, Marcove RC, Jacobs B, Huvos AG (1974) Malignant fibrous histiocytoma and osteosarcoma in association with bone infarct. J Bone Joint Surg Am 56: 932-940. [Crossref]

10. Johnson LC (1953) A General Theory of Bone Tumors. Bull N Y Acad Med 29: 164 171. [Crossref]

11. Bahk W-J, Lee A-H, Kang Y-K, Park J-M, Chung Y-G, et al. (2010) Infarct associated sarcoma: A possible pathogenesis based on histological observation of repair tissue origin in two cases. Acta Oncologica 49: 868-872.

12. Grimer RJ (2005) Surgical options for children with osteosarcoma. Lancet Oncol 6 85-92. [Crossref]

Copyright: (C2021 Spazzoli B. This is an open-access article distributed under the terms of the Creative Commons Attribution License, which permits unrestricted use, distribution, and reproduction in any medium, provided the original author and source are credited. 